\title{
Paralysis after treatment for thyrotoxicosis
}

\author{
HLA Janssen, PHLM Geelhoed-Duijvestijn, AW de Weerd
}

A 41-year-old man was evaluated in February 1994 for weight loss, tremor, tachycardia and goiter. Thyroid function tests and a thyroid scan were consistent with the diagnosis of Graves' disease and he was treated with propylthiouracil $(300 \mathrm{mg} /$ day). Two months later, the patient presented to the emergency room with a flaccid paralysis of all extremeties. At night he had experienced tingling in his legs followed by weakness that ascended to his arms. At the time of admission he was able to move only head and neck. He denied shortness of breath, dysphagia, diplopia or any symptoms suggestive of hyperthyroidism. Except for propylthiouracil he had not used other medication, drugs, alcohol or licorice. The patient was an engineer who had taken refuge from Iraq where he had been in political imprisonment for several years. He had been in The Netherlands for eight months. He was unaware of any family members with neurologic or thyroid disease. On physical examination he had a temperature of $37.2^{\circ} \mathrm{C}$, a pulse rate of 85 beats/ min and a blood pressure of $100 / 70 \mathrm{mmHg}$. There was a diffusely enlarged thyroid gland. Neurologic examination demonstrated an alert and well oriented patient with a flaccid quadriplegia. Deep tendon reflexes were absent and there was no sensory impairment. Results of investigations at admission are listed in box 1.

The patient was admitted to the intensive care unit where potassium was administered. Seven hours after admission his paralysis had disappeared completely and his serum potassium had normalised to $4.4 \mathrm{mmol} / \mathrm{l}$. Because of intractable itch propylthiouracil was replaced by thiamizole $30 \mathrm{mg}$ daily. Four days later, after $\mathrm{KCl}$ supplementation was discontinued, he again experienced severe weakness in all limbs on awakening. The potassium level had dropped to $2.9 \mathrm{mmol} / 1$. After supplementation of $\mathrm{KCl}$, both the hypokalaemia and the paralysis disappeared again with a few hours.

Westeinde Hospital, The Hague, The Netherlands Department of Internal Medicine HLA Janssen PHLM GeelhoedDuijvestijn Department of Clinical Neurophysiology

AW de Weerd

Correspondence to Harry LA Janssen, Department of Internal Medicine, Building 1, C1-R41, University Hospital Leiden, PO Box 9600,2300 RC Leiden, The Netherlands

Accepted 27 June 1996

\section{Investigation results}

- sodium $143 \mathrm{mmol} / \mathrm{l}$, potassium $1.6 \mathrm{mmol} / \mathrm{l}$, chloride $111 \mathrm{mmol} / \mathrm{l}$, bicarbonate $24 \mathrm{mmol} / \mathrm{l}$, calcium $2.19 \mathrm{mmol} / \mathrm{l}$, creatinine $54 \mathrm{mmol} / \mathrm{l}$, glucose $6.6 \mathrm{mmol} / \mathrm{l}$

- free thyroxine $20 \mathrm{pmol} / 1(9-23 \mathrm{pmol} / \mathrm{l})$, triiodothyronine $3.7 \mathrm{nmol} / 1(1.2-3.2 \mathrm{nmol} / \mathrm{l})$, thyroidstimulating hormone $<0.01 \mathrm{mU} / 1(0.2-5 \mathrm{mU} / \mathrm{l})$, cortisol $628 \mathrm{nmol} / 1(180-730 \mathrm{nmol} / \mathrm{l})$, aldosterone $244 \mathrm{pmol} / \mathrm{l}$ supine $(170-610 \mathrm{pmol} / \mathrm{l})$

- potassium in urine $4 \mathrm{mmol} / 1$

- electrocardiography: sinus rhythm with diffuse ST and T wave changes, and the presence of U waves

- electromyography: normal nerve conduction velocities and no evidence for polyradiculopathy

Reference ranges in parentheses

Box 1

\section{Questions}

1 What is the most likely diagnosis?

2 What treatment would you advise in the acute phase?

3 What management would you recommend to prevent further attacks of weakness? 


\section{Answers}

\section{QUESTION 1}

This case illustrates the classical symptoms of thyrotoxic hypokalaemic periodic paralysis (THPP). The emergency physician was unfamiliar with the disease entity and admitted the patient with a differential diagnosis of thyrotoxic myopathy, Guillain-Barré syndrome and hysterical paralysis. After the hypokalaemia became apparent the right diagnosis was made. The differential diagnosis of muscle weakness due to hypokalaemia is listed in box 2 .

\section{QUESTION 2}

Although the acute episode of paralysis usually remits spontaneously, correction of the hypokalaemia with oral or intravenous administered potassium might hasten recovery of muscle function and prevent cardiac arrythmias. Since the total body potassium store is normal, vigorous repletion may result in hyperkalaemia.

\section{QUESTION 3}

Permanent recovery to the euthyroid state and treatment with $\beta$-blocking agents are the best options to prevent recurrence of THPP. Our patient demonstrates that THPP can occur in a subclinical phase of hyperthyroidism several weeks after antithyroid treatment has been started (figure). Further follow-up of this case illustrates that physicians should be extremely cautious with thyroxine replacement for posttreatment hypothyroidism in these patients. After the second episode of paralysis, our patient was treated with propranolol $(120 \mathrm{mg} /$ day) which appeared to be effective in preventing further attacks. However, a few weeks after discharge propranolol and antithyroid treatment were stopped and thyroxin replacement was started because of invalidating fatigue and

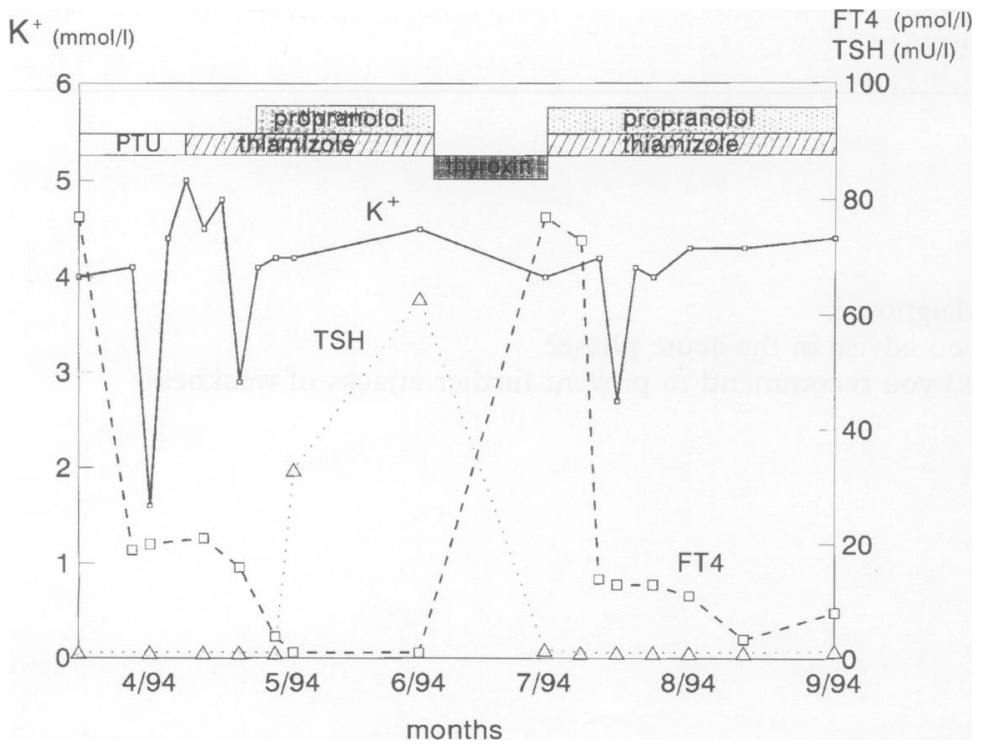

Figure Levels of serum potassium $\left(\mathrm{K}^{+} ;-\right)$, free thyroxine $(\mathrm{FT} 4 ;-\ldots)$ and thyroid-stimulating hormone $(\mathrm{TSH} ; \ldots$ ) during the course of recurrent attacks of hypokalaemic periodic paralysis after the start of antithyroid treatment. Horizontal bars denote treatment with propylthiouracil (PTU; $300 \mathrm{mg} /$ day), thiamizole $(30 \mathrm{mg}$ ) day), propranolol $(120 \mathrm{mg} /$ day $)$ and thyroxine $(50 \mu \mathrm{g} /$ day $)$ biochemical signs of hypothyroidism (thyroidstimulating hormone $63 \mathrm{mU} / 1$; free thyroxine $<2.5 \mathrm{pmol} / \mathrm{l})$. This rather drastic change in the therapeutic regimen was soon followed by a second period of thyrotoxicosis (thyroid-stimulating hormone $0.04 \mathrm{mU} / 1$; free thyroxine $77 \mathrm{pmol} / \mathrm{l}$ ) for which thiamizole and propranolol were restarted. Ten days after the start of this treatment the patient experienced his third attack of periodic paralysis (potassium $2.7 \mathrm{mmol} / \mathrm{l})$. He recovered quickly and on follow-up no further episodes of paralysis were reported.

\section{Discussion}

Periodic payalysis due to hypokalaemia is a rare but dramatic complication of hyperthyroidism. Patients usually present in the emergency room with an overwhelming acute systemic muscular weakness without other neurologic symptoms. Although the association between thyrotoxicosis and hypokalaemic periodic paralysis is well described, establishing the diagnosis can be difficult. ${ }^{1}$ This relates to the rarity of the disease and to the fact that clear manifestations of a thyrotoxic state are often absent during the initial paralytic attack. ${ }^{2}$ The clinical manifestations of THPP include a symmetric flaccid paralysis progressing from distal to central muscle groups mainly involving the lower extremities. The level of consciousness and sensation are unaffected. Attacks mostly occur at night and can be precipitated by ingestion of excessive carbohydrate load, infection, emotional stress and strenuous physical activity followed by rest or sleep.

Patients from East Asia strongly predominate in the ethnic distribution of the disease. ${ }^{3}$ There is usually no familial occurrence, although the disease has a strong male preponderance and it is presumed that a genetic basis is present since patients carrying human leucocyte antigen types A2 Bw22 and Aw19 B17 appear to have an increased relative risk. $^{4,5}$

\section{Causes of muscular weakness due to} hypokalaemia

Intracellular shift of potassium:

- metabolic or respiratory alkalosis

- elevated $\beta$-adrenergic activity

- increased availability of insulin

- thyrotoxic periodic paralysis

- familial hypokalaemic periodic paralysis

- barium poisoning

Real potassium deficit:

- gastrointestinal loss: infectious diarrhoea, coeliac disease, short bowel syndrome, laxative abuse, tube drainage, intestinal fistulas

- renal loss: primary hyperaldosteronism, licorice ingestion, Bartter's syndrome, diuretics, renal tubular acidosis, hypomagnesaemia, ketoacidosis, vomiting, salt-wasting nephropathies, ureterosigmoidostomy 
The cause of the hypokalaemia, which is the cardinal biochemical abnormality during an attack, remains controversial. It is clear that the hypokalaemia reflects a shift of potassium into the intracellular compartment rather than depletion of body potassium stores. Thyroid hormones have been shown to increase number and activity of the $\mathrm{Na}^{+}, \mathrm{K}^{+}$-ATPase channels in skeletal muscles. ${ }^{6}$ This phenomenon is partly related to an increase of $\beta$-adrenergic receptor responsiveness that accompanies hyperthyroidism and which can be treated with propranolol. ${ }^{7}$ Furthermore, in vitro studies demonstrated that patients with THPP respond to thyrotoxicosis with a smaller decrement of $\mathrm{Na}^{+}, \mathrm{K}^{+}$-ATPase activity than thyrotoxic patients without a history of paralysis. ${ }^{8}$ Hyperinsulinaemia, which also activates $\mathrm{Na}^{+}, \mathrm{K}^{+}$-ATPase-induced transport of potassium, appears to be present in many THPP patients and might be another important factor in the precipitation of the paralytic attack. ${ }^{9}$ The hyperadrenergic state and hyperinsulinaemia could explain the provocation of paralysis after physical activity and carbohydrate-enriched meals, respectively. Disturbances in calcium transport which may adversely change muscle excitation-contraction coupling have also been proposed as an aetiological factor of actute paralysis in THPP. ${ }^{10}$

Interestingly, in our patient the episodes of paralysis did not coincide with the severity of thyrotoxicosis. One could question why all attacks occurred after antithyroid therapy was

1 Ober KP. Thyrotoxic periodic paralysis in the United States Report of 7 cases and review of the literature. Medicine 1992 71: $109-20$.

2 Bergeron L, Sternbach GL. Thyrotoxic periodic paralysis. Ann Emerg Med 1988; 17: 843-5.

3 McFadzean AJS, Yeung R. Periodic paralysis complicating thyrotoxicosis in Chinese. BMF 1967; 1: 451-5.

4 Yeo PPB, Chan SH, Lui KF, Wee GB, Lim P, Cheah JS.

4 Yeo PPB, Chan SH, Lui KF, Wee GB, Lim P, Cheah JS

5 Sum CF, Kok ACK, Tan KT, Chan SH, Cheah JS. Human

5 Sum CF, Kok ACK, Tan KT, Chan SH, Cheah JS. Human leucocyte antigen in a Chinese family with thyrotoxic
periodic paralysis in Singapore. BMf 1988; 297: 594-5.

6 periodic paralysis in Singapore. BMF 1988; 297: 594-5. Clausen T. Effect of thyroid function on number of $\mathrm{Na}-\mathrm{K}$ pumps in human skeletal muscle. Lancet 1984; ii: 8-10. instituted and both biochemical and clinical signs of the hyperthyroid state had abated. Although many authors suggest that overt symptoms of hyperthyroidism are usually absent during an episode of periodic paralysis, in the typical case, treatment for thyrotoxicosis leads to cessation of attacks. The development of THPP in the subclinical state of hyperthyroidism argues against a close correlation between levels of thyroid hormones and $\mathrm{Na}^{+}, \mathrm{K}^{+}$-ATPase activity. Several hypotheses could explain the appearance of paralysis in the phase of diminishing hyperthyroidism. First, during severe thyrotoxicosis continuing physical activity without rest may prevent paralytic attacks. Second, it is known that a state of grave thyrotoxicosis is associated with important insulin resistance. The start of antithyroid therapy causes a reduction in insulin resistance which, in the presence of hyperinsulinaemia, may lead to an additional shift of potassium from the extracellular fluid and periodic paralysis. A third possibility, which was not present in our patient, but still may account for continuing hypokalaemic paralysis with normal free thyroxin levels, is a state of triiodothyronine toxicosis. ${ }^{11}$

\section{Final diagnosis}

Thyrotoxic hypokalaemic periodic paralysis

Keywords: thyrotoxicosis, hyperthyroidism, hypokalaemia, paralysis

7 Conway MJ, Seibel JA, Eaton P. Thyrotoxicosis and periodic paralysis: improvement with beta blockade. An Intern Med 1974; 81: $332-6$.

8 Lam KSL, Yeung RTT, Benson EA, Wang C. Erythrocyte sodium-potassium pump in thyrotoxic period paralysis. Aust NZ ₹ Med 1989; 19: 6-10.

9 Lee KO, Taylor EA, Oh VMS, Cheah IS, Aw SE. Hyperinsulinaemia in thyrotoxic hypokalaemic periodic pyperinsulinaemia in thyrotoxic hypd

$10 \mathrm{Au} \mathrm{KS}$, Yeung RTT. Thyrotoxic periodic paralysis: periodic variation in the muscle calcium pump activity. Arch Neurol variation in the musc

11 Sunohara N, Satoyoshi E. Triiodothyronine (T3) toxicosis with hypokalemic periodic paralysis. Eur Neurol 1984; 23 $100-3$ 